\title{
Volt-Ampere Characteristics for Arc Discharge in Low Pressure at Gas $\mathrm{CO2}$
}

\author{
Dr. Abdul Hussain A.Khadyair ${ }^{1}$, Dr.Ahmed Hmeed wanas ${ }^{2}$, Jaafar Lateef Haji ${ }^{3}$ \\ ${ }^{1,2,3}$ (physics Department, College of Eduction/ University Al-Qadisiyah, Iraq country)
}

\begin{abstract}
The research paper discussthe change in current and voltage of arc discharge with the pressure CO2 gas respectively. at gas pressure of (2Torr) to (12Torr), we measured current and voltage at this range of pressures byvarying distances were changing the distance between the electrodesat range $1 \mathrm{~mm}$ to $10 \mathrm{~mm}$ as shown through research that the current and voltage are changing with every change of pressure and the distance.
\end{abstract}

Keywords: Volt, Ampere, discharge arc

\section{Introduction}

Electric arcs are considered thermal plasma, which has many benefits, such as high current high temperature, and the intensity of the high radiation [1] where the amount of current of the order of 1A or more [2] consists arc of the three major regions: the area anode and column plasma and cathode region, electrodes regions (anode and cathode) are formed from the convergence zones between the plasma gas and conductive solids Cloud [3] and the regions of the arc are shown in Figure 1, has been generating this type of discharge industrially "since early last century, and that period beganResearch evolution to improve its applications [4] There are many applications of the arch is used in broad areas such as spraying plasma cutting, lighting and treatment of toxic waste, cutting, welding and other [5] and many studies have been conducted to verify the effect of different conditions, such as gas factor and materials-electrode, forms and application a magnetic field on the arc and complex interactions between the plasma and the electrodes properties (or cool the walls) [6] where the researcher Xiu and his group studied electric arc dc defect experimental study and clarify a defect where the various tests to determine the effect of various factors on the arc likeThe length of the interval between the electrodes, and current and others have noted that the voltage arc increases with the gap [7] The researcher Zhao Peng and his group studied plasma arc characteristics of nitrogen with Anode molten he used in his study of a cathode made of graphite and testing voltage as a current function and plasma along virtual noted that the voltage increases nonlinearly with increasing plasma along the virtual [8].

\section{Governing equations}

We have explained in the preceding paragraph that the arc consists of three main areas which the anode and the plasma column and the cathode region and will focus on the plasma column area where we assume that it local thermodynamic equilibrium (LTE) the equations for the conservation of mass, enthalpy, radial momentum are respectively [9]:

$$
\begin{gathered}
\frac{1}{r} \frac{\partial}{\partial r}\left(r \rho v_{r}\right)+\frac{\partial}{\partial z}\left(\rho v_{z}\right)=0 \\
\frac{\partial}{\partial r}\left(\rho v_{r} h\right)+\frac{\partial}{\partial z}\left(\rho v_{r} h\right)=\frac{1}{r} \frac{\partial}{\partial r}\left(\frac{r k}{c_{p}} \frac{\partial h}{\partial r}\right)+\frac{\partial}{\partial z}\left(\frac{k}{c_{p}} \frac{\partial h}{\partial z}\right)+\frac{j_{r}^{2}+j_{z}^{2}}{\sigma}-U \\
\rho\left(v_{r} \frac{\partial v_{r}}{\partial r}+v_{z} \frac{\partial v_{r}}{\partial z}\right)=-\frac{\partial p}{\partial r}-j_{z} B_{\theta}+\frac{1}{r} \frac{\partial}{\partial r}\left(2 r \eta \frac{\partial v_{r}}{\partial r}\right)+\frac{\partial}{\partial z}\left(\frac{\eta \partial v_{z}}{\partial r}+\frac{\eta \partial v_{r}}{\partial z}\right)-2 \eta \frac{v_{r}}{r^{2}}(3) \\
\rho\left(v_{r} \frac{\partial v_{z}}{\partial r}+v_{z} \frac{\partial v_{z}}{\partial z}\right)=-\frac{\partial p}{\partial z}+j_{r} B_{\theta}+\frac{\partial}{\partial z}\left(2 \eta \frac{\partial v_{z}}{\partial r}\right)+\frac{1}{r} \frac{\partial}{\partial r}\left(\frac{r \eta \partial v_{z}}{\partial r}+\frac{r \eta v_{r}}{\partial z}\right)+\rho g .
\end{gathered}
$$

The material functions are the enthalpy $h$, specific heat $\mathrm{C}_{\mathrm{p}}$, Thermal conductivity $k$, viscosity $\eta$, electrical conductivity $\sigma$, plasma density $\rho$, radiation emission coefficient $U$, all of which are function of temperature for the ambient gas pressure of the arc. Physical conditions are properties by temperature $\mathrm{T}$, pressure $\mathrm{p}$, either $v_{r}$ are pivotal velocity, $v_{z}$ represents the are functions of the radius $r$ and the site axial $z$ in equations (1) - (4), and g represents accelerate gis the acceleration due to the gravity.The current density $\mathrm{j}$ and the magnetic field $\mathrm{B}$ in the previous equations we can get it by using the current continuity equation and one of Maxwell's equations:

$\frac{1}{r} \frac{\partial}{\partial r}\left(\mathrm{r} \sigma \frac{\partial \emptyset}{\partial r}\right)+\frac{\partial}{\partial z}\left(\sigma \frac{\partial \emptyset}{\partial z}\right)=0$

$$
j_{r}=\sigma \frac{\partial \emptyset}{\partial r} j_{z}=\sigma \frac{\partial \emptyset}{\partial z}(6)
$$




$$
\frac{1}{r} \frac{\partial}{\partial r}\left(r B_{\theta}\right)=\mu_{o} j_{z}
$$

Where $\emptyset$ is the electric potential and $\mu_{o}$ is the permeability of free space [10].

\section{Experimental Set-Up}

It has been using the experimental system shown in Figure (2) to study the properties of voltage -current of arc, where this system consists of a discharge arc chamber and devices used

\section{3-1 discharge arc chamber}

This part of the: consists flange upper Made Brass outer diameter of $17.5 \mathrm{~cm}$ and external thick $4.38 \mathrm{~cm}$ this flange contains a set of holes, including for the purpose of the gas flow including the feed cathode voltage is including thermocouple inside the chamber, and also "contains holes through which water flows either side for the purpose of cooling, while the cylindrical glass used are $15 \mathrm{~cm}$ in diameter and $14.1 \mathrm{~cm}$ length of about $5 \mathrm{~mm}$ thick and made of Pyrex glass it compartment components discharge arc lower flange made of Brass has a central hole for the purpose of supplying the anode voltage is changed and the distance between the poles through the rod Brass coaxial, and cathode user is shaped cylindrical and conical end so that the top of the cone angle is approximately $60^{\circ}$ The use of four of the cathode materials for the manufacture of an iron, stainless steel, Brass, copper and Figure 3 shows the image of the cathodes used and made of different materials The anode was a variety of copper almost diameter disc body " $8.6 \mathrm{~cm}$ and $1.8 \mathrm{~cm}$ fish. And Figure 4 shows the image to the user Anode.

\section{3-2- Devices used}

Was used dump mechanical pump (Rotary Pump) type TRIVAC D16B , pump maximum value is 102Torr it is suitable and we got unloading rate of up to about 10-2Torr and then gas is pumped Alarcon to get the desired compression section use pressure type pirani scale which can measure the pressure of 10-2 torr to 20Torr is appropriate to our experience. The measurement of the voltage between the cathode and the anode using a digital Avometer is set to measure the DC Voltage (200V- 0V), And we used Lemaitre Digital can currents up to 400A equipped .we used power supply gives high current of INGCO company, Over the currents they provide $10-200 \mathrm{~A}$, and It is suitable for working conditions.

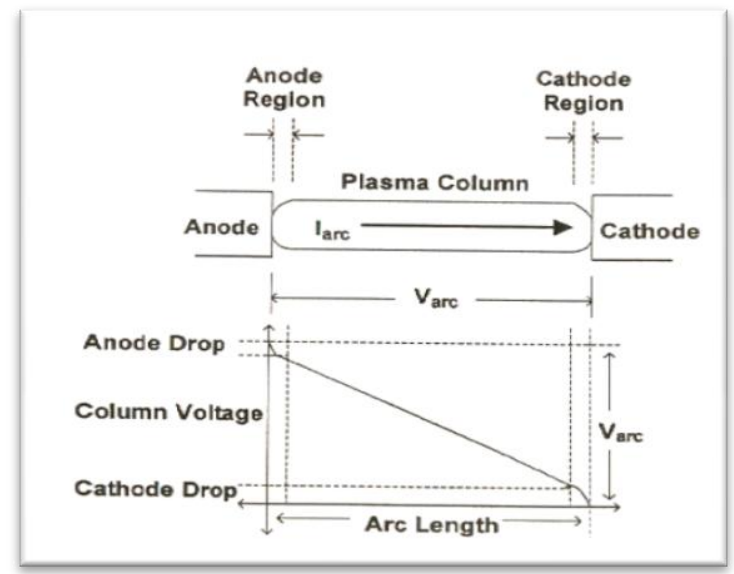

Figure( 1) electric arc properties[11]

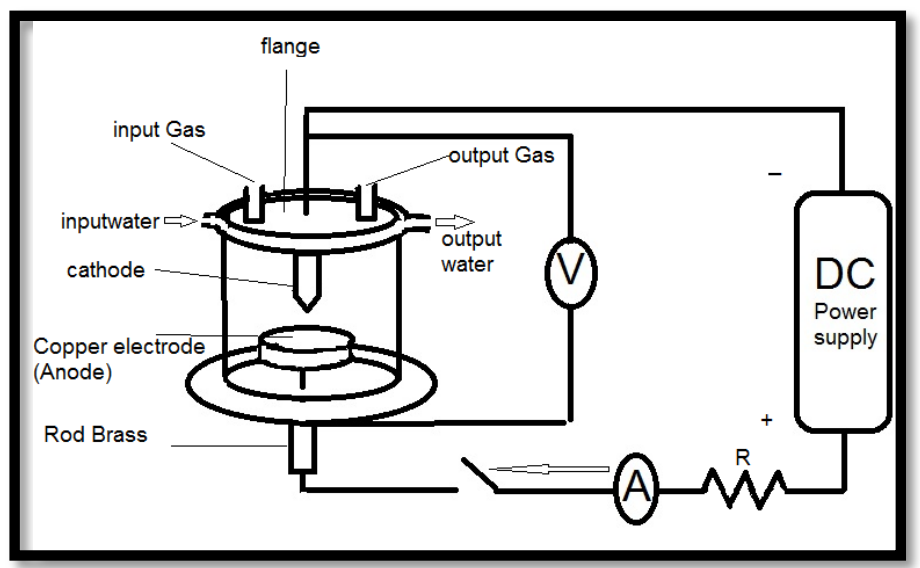

Figure 2 scheme to arc discharge system 


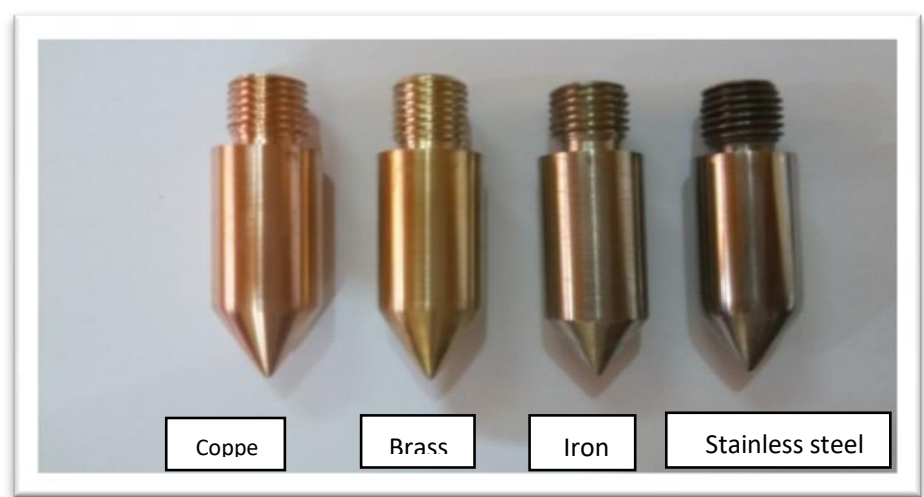

Figure 3 pictures of a cathode made of different materials

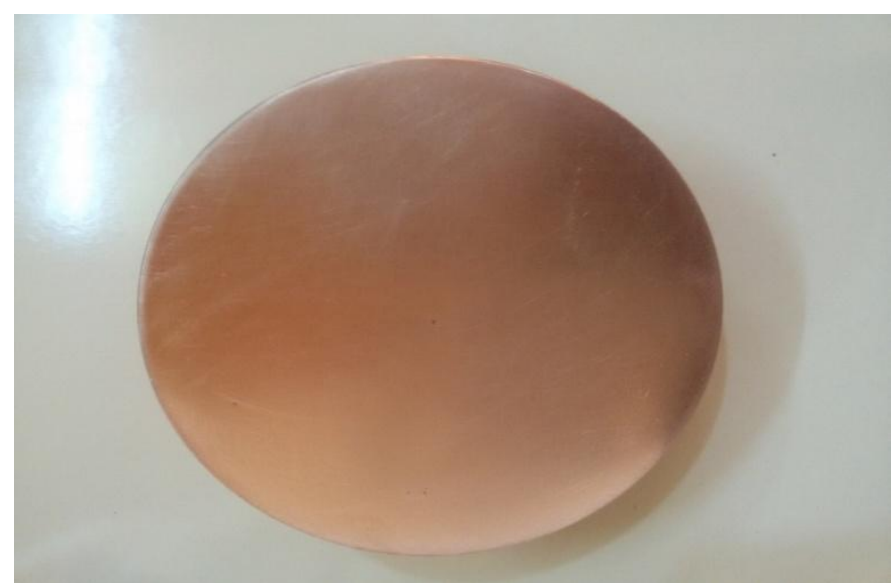

Figure 4 picture of the user Anode

\section{The experimental conditions}

We found that the cathode after a number of electrical discharges begins to melt in part, "which we have to resample the same body as the tip spire begins to melt and change its shape, we used electrical a machine Edging with Quicken variable installed on the table and we consider certain types of tablets Edging for that machineAs well as the anode, it is influenced by vacuum arc, where we read the part of the sediment generated by the fusion of the cathode accumulate on the anode, which requires toremovedof the sediment, and after the re-formation of the cathode we use sandpaper coarse leaves and then soft, then softer and is then washed with alcohol center by $96 \%$, While the glass cylinder and found that it also "be affected by sediment resulting from the discharge and sometimes is volatile spray of molten cathode on the inner surface of the cylinder, we are removed of the sediments we used sandpaper sheets carefully to remove those sedimentsThe cooling process of the upper flange has used the water pump in the cooling process of the upper flange, by connecting the pump Plastic tubes you enter cold water to the upper flange connecting through holes within them and thus cooling the cathode and Figure 5 shows pictures of the cathode and anode and cylindrical bottle after discharge arc process.

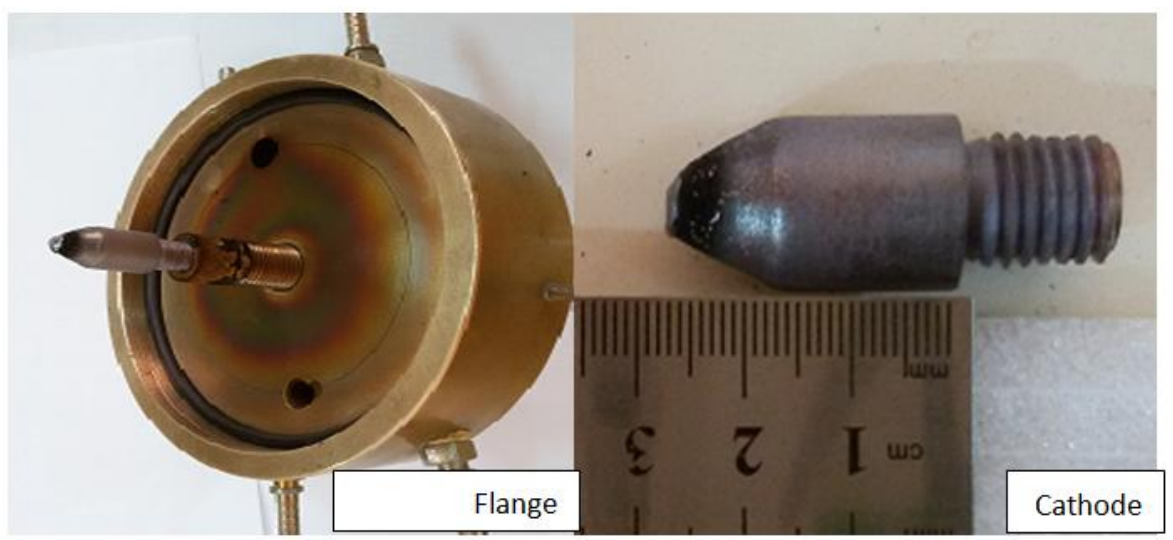



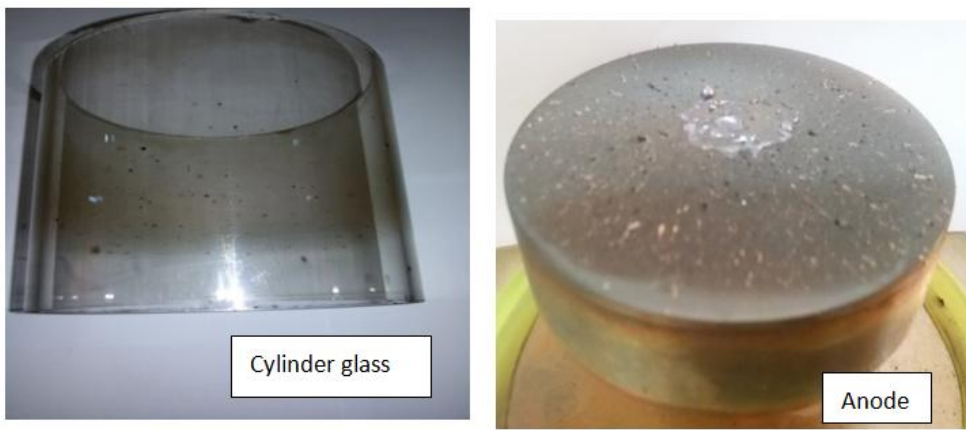

Figure 5 shows pictures form the cathode and the anode and the cylinder glass after each arc discharge

\section{5-steps of work}

1-Electric arc discharge chamber from the air to the pressure (0 torr)

2-It has been set distance between the electrodes using the ruler Metric

3-Ar gas is leaking into his lap arc discharge

4-Steps 1 and 3 twice returned for the purpose of obtaining better conditions purity of the gas inside the chamber, and then adjust the gas pressure

5-Start running the water pump special upper flange

6-burn the arc by draw method

7-Are recording current flowing, voltage, pressure and current which equip Source

8 -Increase the arc current and record voltage for several values of the current-

9-Reshape the cathode and the removal of sediment generated from the arc discharge

10-Returned steps 1 to 8 for each type of cathode materials

\section{Results and Discussion}

In the figures $6,7,8,9$ we obtain the voltage and current reading and Change them with the pressure and the distance between the poles and the various cathode materials and we have noticed that the current increases with increasing pressure due to the increasing the number of collisions leading to an increase of electrons freed and also noticed by increasing current with the distance between the electrodes wenotice increase free path of the electron and increase the kinetic energy we noticed that the amount of voltage as well as the amount of current oscillates rapidly between the landing and the height where we take the average of all the readings we have recorded which show us the volatility in the reading caused by the gas flow which affects the stability of the arcas well as showing us that this volatility is due to the melting of part of the cathode, an issue again cause a lack of stability of the arc as the arc oscillates rapidly around the axis, and noted that the increase in gas pressure and the distance between the electrodes voltage causes an increase due to increased resistance to arc and this is consistent with the researcher [8]. as well as we have noted be tight for the cathode formed from iron and stainless steel that the amount of change in the current and voltage with the change of the gas pressure and the distance between the poles as well as the results will be close for the cathode formed from copper and Brass This may be the cause convergence in the results is due to the convergence of the work functions of iron and stainless steel, as well as the convergence of the work functions of copper and Brass.

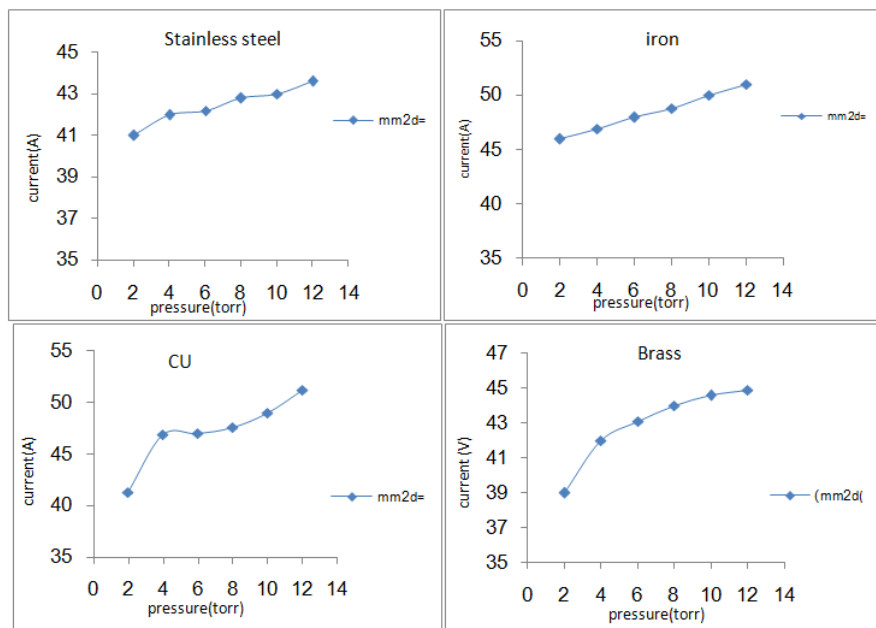

Figure 7 shows the relationship between the pressure and the power of $\mathrm{CO}_{2}$ gas and all the electrodes used 


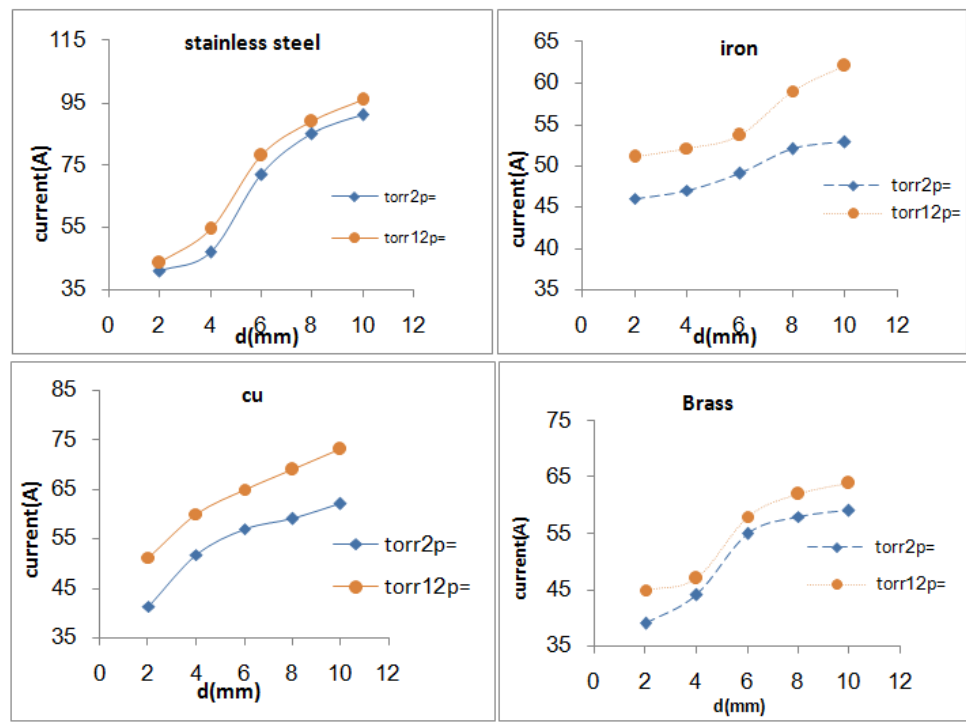

Figure 8 shows the relationship between the current and the distance between the electrodes of all the electrodes used for the $\mathrm{CO}_{2}$ gas
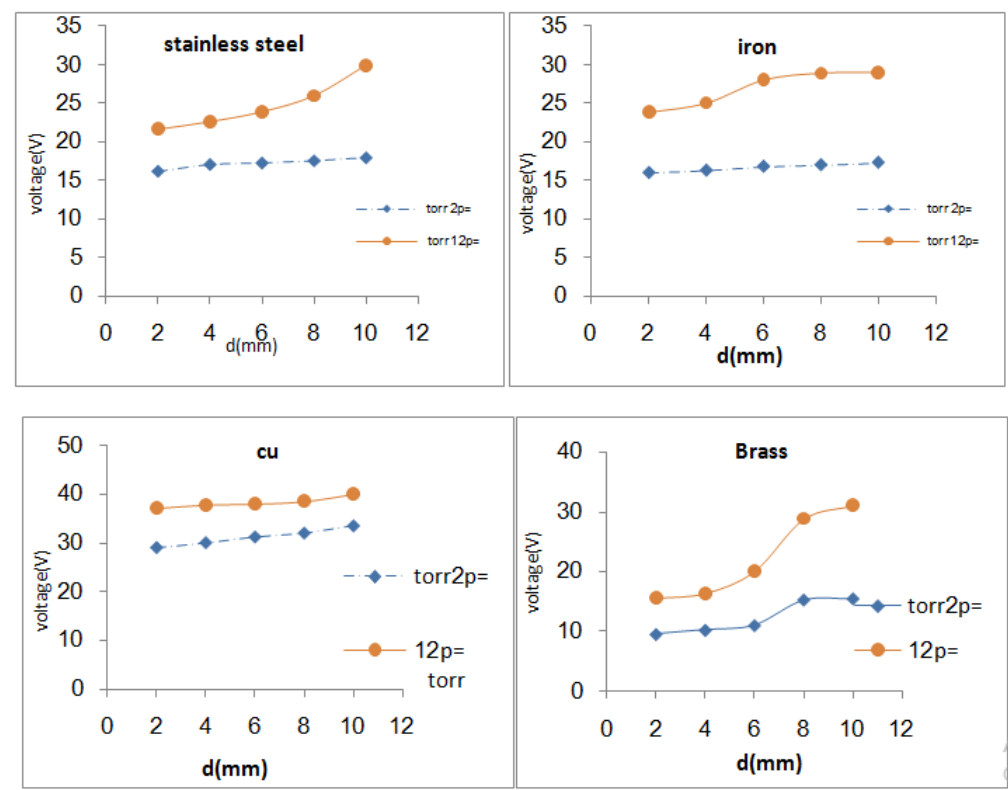

Figure9 represents the relationship between the distance between the electrodes and the voltage for all polar used for $\mathrm{CO}_{2}$ gas

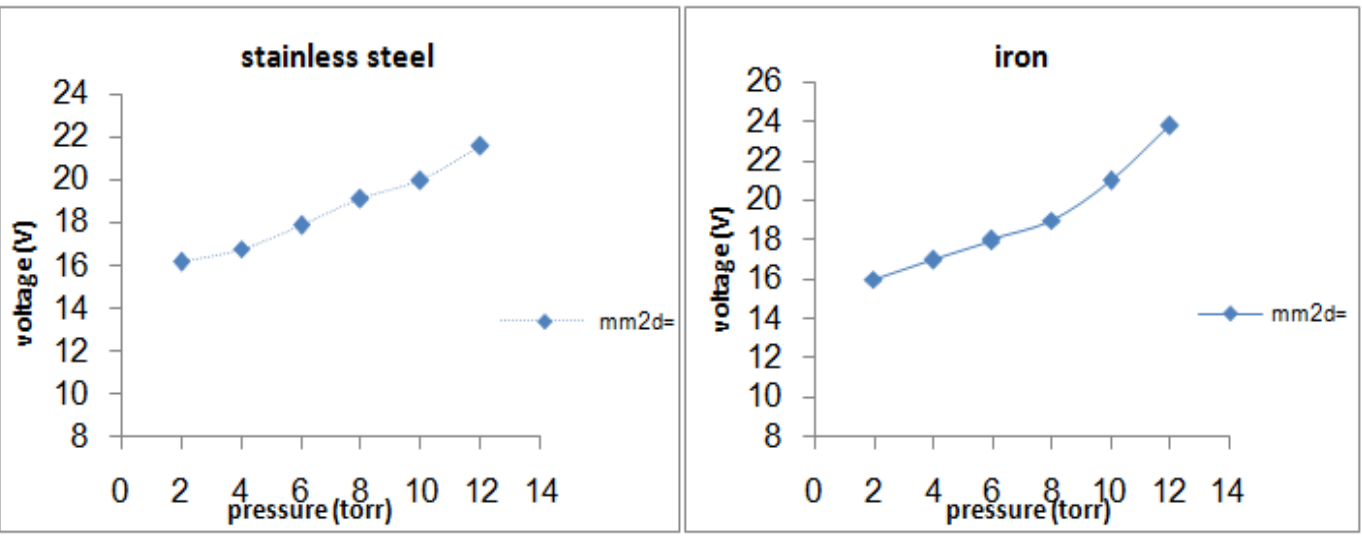



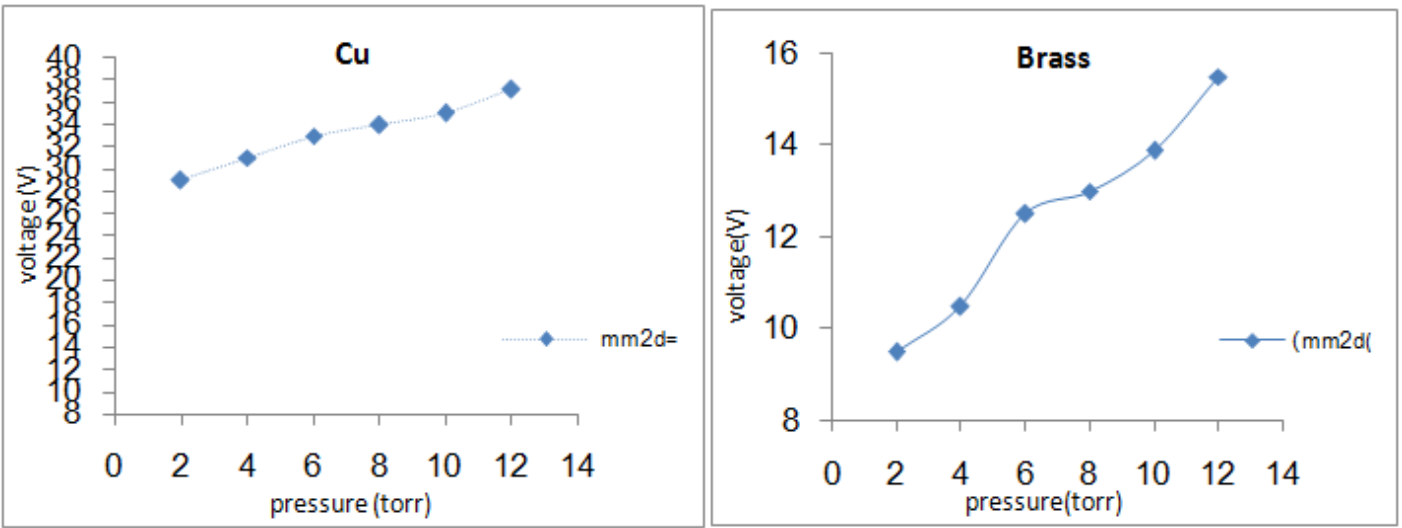

Figure 10 represents the relationship between pressure and voltage different electrodes of $\mathrm{CO}_{2}$ gas

\section{References}

[1]. Zhao. P, Ni.Guo-Hua, Jian .Y M, and Chen L.W 2010, J,Hazard.Mater, 181, 580

[2]. Yuri P. Raizer, 1991Gas Discharge Physics, Springer,

[3]. J..M. Somerville, 1959 "The Electric Arc" London , U.K.: Wiley,

[4]. X. Yau, L. Herrea, Y.Huang, and J.Wang2012The Delection of DC Arc fault: Experimental study and fault Recognition, IEEE 978$1-4577-1216$

[5]. He-P.Li, M.S. Benilov 2007, J.Phys. 40 2010-2017

[6]. Gidalevich E, Goldsmith S and Boxman R L 2004 Plasma Sci. Source Sci. Technol. 13 454-60

[7]. PeiuanZut, J JLowke and R Morrow, 1992 A unified theory of free burning arcs Cathodesheaths and cathodes, J. phye. D: phys. 25

[8]. Z. Peng, N. Guo-Hua, M. Yue-Dong, N Masaaki 2013. Volt- Ampere characteristics of a nitrogen DC Plasma arc with anode melting.Chin .Phys.B, vol.22, No.6

[9]. Loweke J J, Kovitya P, and Schmidt H-P 1992 J. Phys. D: appl Phys. Sumitted

[10]. Tanaka M, Terasaki H, Ushio M, and Lowke J J 2003, Plasma chm.Plasma Process. 23585.

[11]. T.E.Browne, Jr. .1955, The electric arc as a circuit element, J. Electrochem. Soc .,vol.102, no.1,pp.27-37, Jan 